\title{
Constructed amphiphilic gel as natural auxiliary medium to mimick remineralized dental hydroxyapatite
}

Kun Tian
1a
Quanyuan Liu
Qin Du $\mathrm{Du}^{1 \mathrm{f}_{*}}$

${ }^{1}$ Department of Stomatology, Hospital of University of Electronic Science and Technology of China (UESTC) \& Sichuan Provincial People's Hosptial, Chengdu, 610071, China

${ }^{2}$ Department of Medical Statistics, West China School of Public Health, Sichuan University, China

${ }^{3}$ School of Medicine, University of Electronic Science and Technology of China (UESTC), Chengdu, 610071, China

IThe two authors, Kun Tian and Yuanyuan Liu contributed equally to this work aemail: tiankun78@hotmail.com, bemail: 3450357@qq.com, cemail: xiaoyun936954705@qq.com demail: 1072718910@qq.com, eemail: 35338551@qq.com, *femail: dududicn@163.com

Keywords: Hydroxyapatite, N-methylene phosphonic chitosan, reconstruction

\begin{abstract}
For obtain enamel-like hydroxyapatite, we are trying to induce the growth of crystal with modified chitosan which can simulate the organic template like extracellular matrix--Amelogenin to induce the deposition of miner ions. Phosphorous acid modification was applying into natural polycation polysaccharide chitosan to synthesize polyanion derivate phosphorylated chitosan. Amphiphilic hydrogels combined with phosphorylated chitosan (+cation) and gelatin (-anion) were built through peptide binding reaction which using Genipin as crosslinker. The gels self assembled on the inert surface of tooth which stimulated by ultraviolet radiation. $\mathrm{CaCl}_{2}$ and $\mathrm{Na}_{3} \mathrm{PO}_{4}-12 \mathrm{H}_{2} \mathrm{O}$ solutions provided mineralized ion then the hydroxyapatite assembled and grown in situ of the tooth. All of the samples detected by FTIR, SEM and XRD to confirm the character of neonatal crystal. The amphiphilic gel can enhance the proportion of organic elements on the surface of dentin which confirmed by FTIR. After mineralized reaction, we observed bunch and column (15-25nm) crystal arranged on the dentin base. XRD result showed the neonatal crystal is hydroxyapatite and scratch tester testified the coalescent force between 22-25N. The amphiphilic gel can be induced the enamel-like crystal grown under demineralizing tooth basement.
\end{abstract}

\section{Introduction}

Enamel is the exterior layer of human tooth and a highly mineralized biomaterial with significant resilience that protects the human tooth from physical and chemical damages [1]. The basic component of mature enamel is hydroxyaptite (HAP) and Fluor-hydroxyapatite (FHAp), it is highly ordered structural organization which fundamental units are rod and inter-rod woven by parallel crystals boundless[2]. As adult tooth enamel is not living tissue, it can hardly remineralize after the substantial mineral loss. Thus, the synthesis of enamel through biomimetics is of great interest to dental clinicians. The key of remineralization or regeneration of tooth hard tissue is finding an appropriate organic template and inducing the process of mineralization. The frequently used agents are fluoride [3], salivary proteins [4], casein phosphopeptide amorphous calcium phosphate [5], or organic polymers [6]. However, these approaches either call for too severe conditions to apply in clinics, or reconstruct structures unlike natural enamel.

The formation of Calcium matrix proteins in bones (mainly collagen type I) and teeth (amelogenin) are associated with the nucleation and growth of hydroxyapatite crystals [7]. For mimicked the physiological course to obtain neonatal hydroxyapatite we chose phosphonic chitosan as the crystal growth monolayer. 
Chitin, a naturally abundant mucopolysaccharide, and the supporting material of crustaceans, insects, etc., is well known to consist of 2-acetamido-2-deoxy-b-D-glucose through a $\beta(1,4)$ linkage [8]. Chitosan, obtained by alkaline N-deacetylation of chitin, (Fig. 1) is one kind of the most widely used natural cationic polysaccharides. Although it is insoluble at physiological $\mathrm{pH}$, but still shows particularly high biocompatibility and fairly low cytotoxicity [9].

Reports reveal that apatite-chitosan composite would be a candidate for a bioactive material. When a functional group e.g., carboxyl group that is effective for apatite nucleation, is present on the surfaces of carboxymethylated chitin and gelatin gum gels, then apatite spontaneously forms on these surfaces [10] [11]. Chemical modification of chitosan including acylation, carboxylation, hydroxylation, etherification, Schiff base reaction, N-alkylation, esterification and hydrolysis, oxidation, halogenation and other graft modification. Researchers attaches great importance to phosphate esterification reaction for phosphate group were induced which have special affinity with calcium ions.

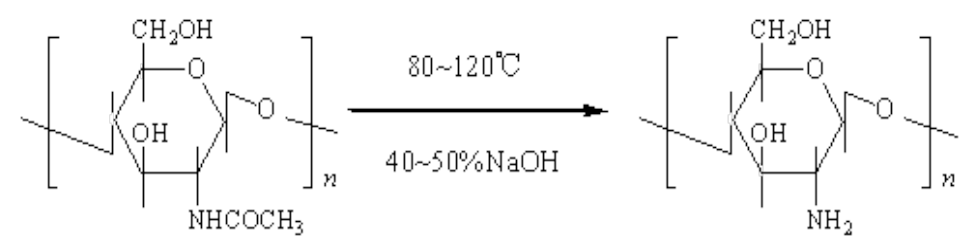

Figure 1. Structure of Chitin and Chitosan

Based on the basic theory, we use phosphonicchitosan molecules model to induce the crystallization of hydroxyapatite to synthesized tooth-like calcium phosphate/hydroxyapatite with $3 \mathrm{D}$-structure in a controllable way in vitro.

\section{Materials and methods}

\section{Phosphonized chitosan}

One equivalent of phosphorous acid in water was added dropwise to one equivalent (w/w) of $2 \%$ chitosan solution in acetic acid with continuous stirring for $1 \mathrm{~h}$. Raised the temperature to $70{ }^{\circ} \mathrm{C}$ and one part of 36.5\%(w/w) formaldehyde was added drop-wise over $1 \mathrm{~h}$ with reflux and heating for 6 h. After dialyzed against demineralized water for $48 \mathrm{~h}$, frozen and freeze-dried finally [12]. Figure 2 showed the reaction of phosphonized chitosan. The variation elements on the surface of sample were investigated by Fourier transformed infrared spectroscopy (FTIR, EQUINOX 55, Bruker Optics, Switzerland).

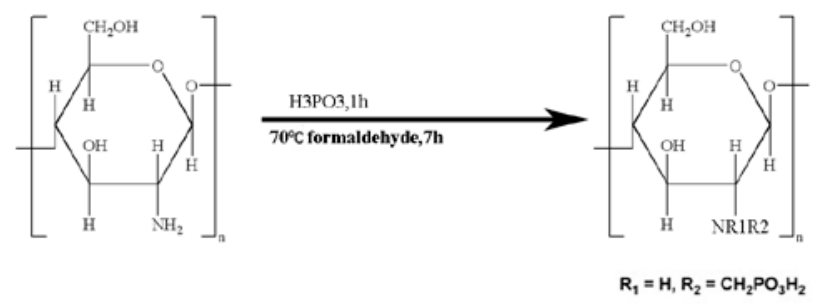

\section{Sample preparation}

Figure 2. Synthesis of $\mathrm{N}$-methylene phosphonic chitosan

Human molars were sliced into $1 \mathrm{~mm}$ thickness disks and etched with 0.5N EDTA to reveal the different orientations of the dentinal tubule. The disks were immersed in 0.02M Tris-HCL buffer (pH7.2,) after cleaned by ultrasonic. The samples were studied by scanning electron microscopy (SEM) (S-2460N, Hitachi, Tokyo, Japan).

\section{Phosphorylation of tooth surface}

The molars disks were daubed with phosphate ions dental adhesive agent equably (Prime\& BondNT, DentsplyDetreyGmbh). The solidification process induced by curing light is $10 \mathrm{~s}$ at least. $\mathrm{N}$-methylene phosphonic chitosan solution(test group) and chitosan solution(contrast group) were daubed to disks of molar after phosphorylation. 


\section{Mineralization in vitro}

Biomimetic calcification solution with $2.58 \mathrm{mM}$ calcium $\left(\mathrm{CaCl}_{2} \cdot 2 \mathrm{H}_{2} \mathrm{O},>74.4 \% \mathrm{CaCl}_{2}\right)$ and $1.55 \mathrm{mM}$ phosphates $\left(\mathrm{KH}_{2} \mathrm{PO}_{4},>99 \%\right)$ at $37^{\circ} \mathrm{C}$, buffered by $50 \mathrm{mM} \mathrm{pH}=7.6$ trihydroxymethylaminomethane (Tris) - hydrochloric acid and $180 \mathrm{mM} \mathrm{NaCl}$. The calcification solution $\mathrm{pH}$ was adjusted by $1 \mathrm{M} \mathrm{HCl}$ to 7.60 using a Metrohm $718 \mathrm{pH}$-STAT. [13]. All the samples were studied by X-ray diffraction (XRD, X'Pert Pro MPD, Philips, Holland), SEM.

\section{Determination of coalescent force}

The coalescent force between tooth surface and neonatal crystal Scratching Method(WS-92 scratch tester). The loading range between $0.01 \mathrm{~N}-30 \mathrm{~N}$, then automatic continuous loading in the following way: trial is conducted initially in precision $0.1 \mathrm{~N}$, scratch speed $2 \mathrm{~mm} / \mathrm{min}$, loading rate for $10 \mathrm{~N} / \mathrm{min}$,loading pressure head is cone Angle $120^{\circ}$ of the diamond, and the tip radius is $0.2 \mathrm{~mm}$.

\section{Results and discussion}

\section{Characterization of CS and NMCS}

FTIR spectrogram showed organic compounds were grafted to the surface of tooth section. Figure 3 showed the FTIR spectrum of chitosan(CS) and N-methylene phosphonic chitosan(NMCS). $894 \mathrm{~cm}^{-1}$ and $1154 \mathrm{~cm}^{-1}$ absoprtion band were the characteristic band of glycosyl in chitosan. Compared with CS, the NMCS spectrogram showed weak band of $-\mathrm{NH}_{2}$ in $1600 \mathrm{~cm}^{-1}$ wave area and sturdy band of acidamideI in $1635 \mathrm{~cm}^{-1}$ and acidamide II in $1524 \mathrm{~cm}^{-1}$. The stretching vibration peak of alcohol hydroxyl lower in1000 100 $\mathrm{cm}^{-1}$, and new vibration peak of $\mathrm{PO}_{4}$ were observed in $1048 \mathrm{~cm}^{-1}$. There were broad absoprtion band between $2000 \sim 3500 \mathrm{~cm}^{-1}$ that suggest dissociative $\mathrm{H}^{+}$in $-\mathrm{NH}_{2}$ were insteaded by $-\mathrm{CH}_{2}-\mathrm{PO}_{3}$.

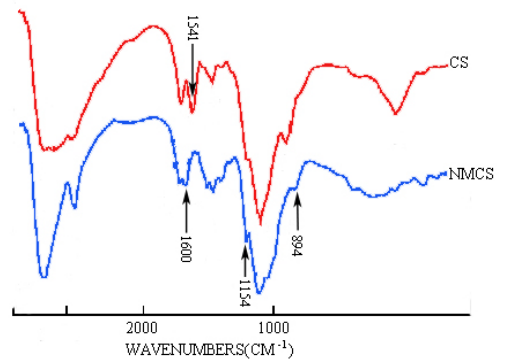

Figure 3. FTIR spectrum of CS and NMCCS

\section{Calcium Phosphate Formation on tooth}

After aging in SCS for four hours, SEM micrographs showed that the enamel rods were blocked by neonatal hydroxyapatite layer. Fig 4(A) showed the structure of enamel rod after decalcificated of EDTA 1h. Type II etching pattern, where the prism boundary is preferentially eroded. Continuous hydroxyapatite layer were formed within 24 hours, and a crystal shape were observed on test groups which NMCS covered the surface of enamel(Fig 4(B)). The mean diameter of the rod was $15 \pm 6 \mathrm{~nm}$. Type I pattern with the prism core preferentially eroded were showed in Fig 4(C). In this section the neonatal hydroxyapatite were mutual parallel arrangement in the interspace after eroded(Fig 4(D)). The habit and size of the crystals were similar to those crystals obtained in prism boundary eroded section.

In contrast group which without chitosan crosslink to enamel after daubed with phosphate ions dental adhesive, only sheet and irregular neonatal apatite could be observed. There were about $2 \mu \mathrm{m}$ columned precipitation of mineral crystals formed on the surface within 24 hours (Fig2(E)). On the surface of tooth, hollow tubules of enamel were only filled in by shot prismatical crystal dispersedly and irregularly(Fig 2(F)). Furthermore, both test group and contrast group have a continuous structure of columns crystal with size of $10-20 \mathrm{~nm}$. 

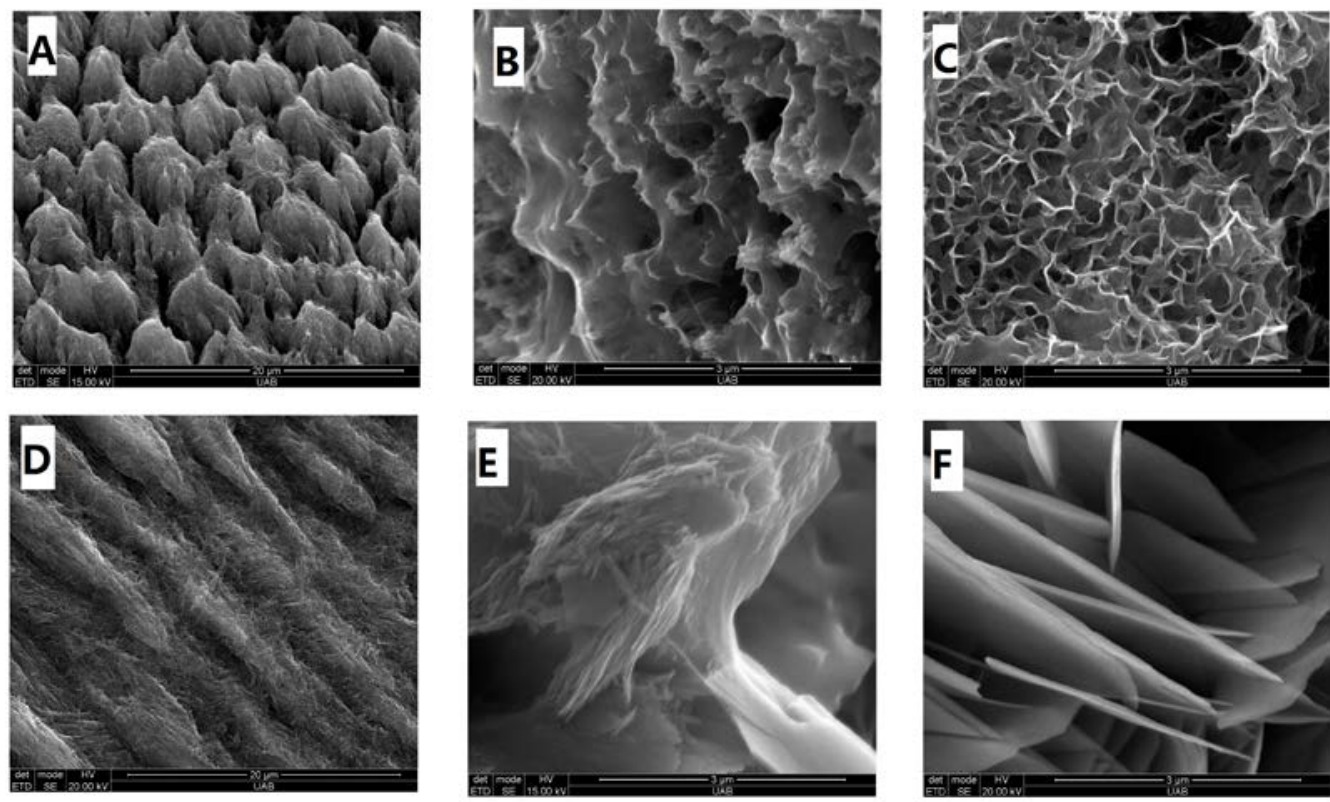

Figure 4. SEM images of neonatal crystal

A.normal enamel rod after decalcificated $24 \mathrm{~h}($ type II). B.the form of crystal after mineralization in test group $48 \mathrm{~h}$ (type II). C.normal enamel rod after decalcificated $24 \mathrm{~h}$ (type I). D.the form of crystal after mineralization in test group $48 \mathrm{~h}$ (type I). E.the form of crystal after mineralization in contrast group $48 \mathrm{~h}$ (typeII). F. the form of crystal after mineralization in contrast group 48 h(type I).

\section{XRD spectrum}

XRD spectrum of the neonatal crystal (Figure 5) suggested that several diffraction peaks around $2 \theta=32^{\circ}$ which are corresponding to the expected Bragg peaks for hydroxyapatite, implying that HA crystals were formed. In test group, the peaks around $25-26^{\circ}$ were sharper than contrast group, and peaks around $42-47^{\circ}$ were smoother than others(Figure 5). This may came from the plentiful organic molecules on the surface of experiment groups which induced the growth of hydroxyapatite and didn't hydrolyze on time.

Test group shows sharper characteristic peak than contrast group in $2 \theta=$ $26^{\circ}, 29^{\circ}, 32^{\circ}, 39^{\circ}$ which corresponding to $\mathrm{Ca}_{10}\left(\mathrm{PO}_{4}\right)_{3}\left(\mathrm{CO}_{3}\right){ }_{3}(\mathrm{OH})_{2}, \mathrm{Ca}_{10}\left(\mathrm{PO}_{4}\right)_{5} \mathrm{CO}_{3}(\mathrm{OH}) \mathrm{F}$, $\mathrm{Ca}_{10}\left(\mathrm{PO}_{4}\right)_{6}(\mathrm{OH})_{2}$ and $\mathrm{Ca}_{10}\left(\mathrm{PO}_{4}\right)_{5} \mathrm{CO}_{3}(\mathrm{OH}) \mathrm{F}$.

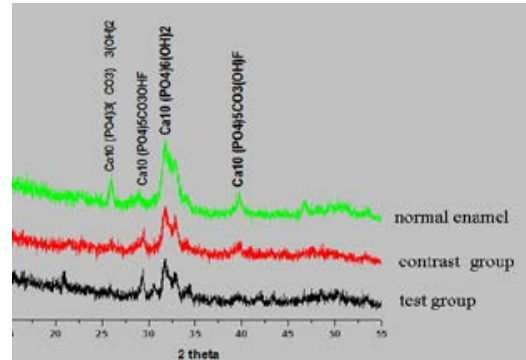

Figure 5. XRD of precipitation formed on tooth surface

Determination of coalescent force

Figure 6 and 7 showed the coalescent force between tooth surface and neonatal crystal in contrast group and test group are 13 15N and 22 25N. 

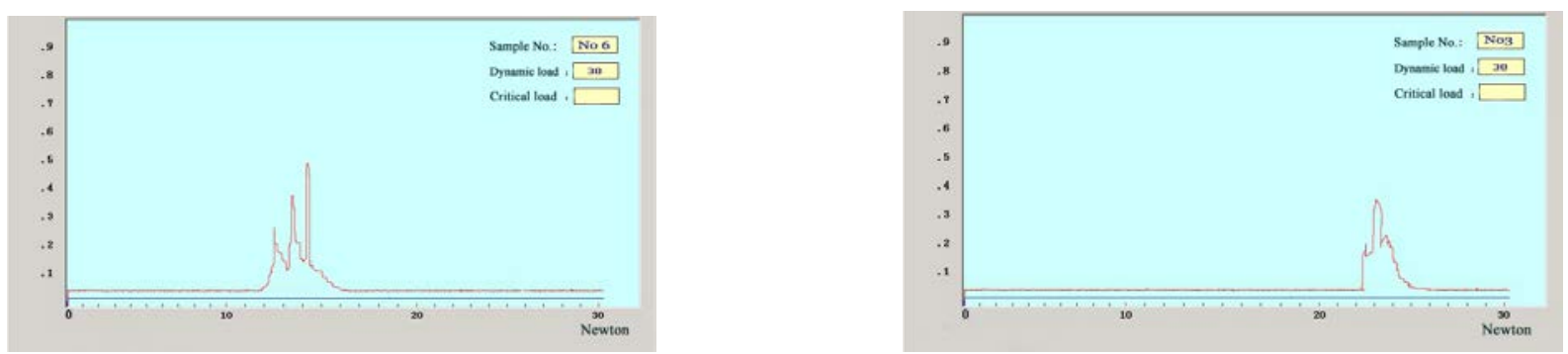

Figure 6. Coalescent force between neonatal crystal and tooth surface in contrast group(CS).

Figure 7. Coalescent force between neonatal crystal and tooth surface in test group(NMCS).

\section{Discussion}

Although dentin and bone are similarly composed of about 70\% hydroxyapatite and 30\% organic material [14], histological analyses demonstrate distinct cellular and morphological differences between these tissues. That's why we chose N-methylene phosphonic chitosan instead collagen to induce the synthesis of Hydroxyapatite which full of phosphate group has special affinity with calcium ions. It should be pointed out that we have tried to deposit the $\mathrm{PO}_{4}{ }^{3+}$ onto different substrates, only the iron-plate substrateis able to produce the enamel-like films. The others may support the growth and orientation of the HA crystals but do not produce the closely aligned prismlike structure. The reason for this is still under investigation.

There is an interesting phenomenon that the eroded pattern would decide the direction of the neonatal crystal(Fig 4 (B)(D)). Acid attack initiated at the rod sheath space then penetrated into the rod core before extending into neighbouring rods through the rod tail [15]. So in the further study the time of eroded and the variety of acid etching agent such as EDTA and $37 \%$ phosphate etc would be observed.

Phosphate ions dental adhesive agent is polyanion organic molecules which not only inducted the demineralization on surface of tooth, but also adsorbed on enamel through calcium phosphate chemical bond. The phosphate groups and halogenated phosphoric acid would bonding to $\mathrm{Ca}^{2+}$ on tooth surface by coordinate bond or formed hydrogen bonds and intermolecular forces with collagen [16]. So dentin adhesive agent can intake calcium and phosphorus from the SCS, then generation apatite is induced.

The successful growth of enamel-like HA under the guide of NMCS not only shows that they are biocompatible, but also shows that under the control of the appropriate signaling molecules and cell-culture conditions it might be possible to induce dentin and pulp formation on the synthetic enamel layer in vitro. The key to specificity in controlled crystallization is the "molecular recognition" effect at the interface between functional groups on the organic macromolecules and ions in the surface of a crystal nucleus [17-18]. The organic model dental collagen-biologic Nmethylene phosphonic chitosan connected by peptide bond and the - $\mathrm{COO}^{-}$headgroup on the peptide can accumulate $\mathrm{Ca}^{2+}$ and $\mathrm{PO}_{4}{ }^{3-}, \mathrm{OH}^{-}$ions and build the beneficial lattice configuration for the plane of hydroxyapatite [19]. Overall, the amphiphilic gel combined with the implementation of $\mathrm{Ca}^{2+}$ in the solution which provide three dimensional sites for directed self-assembly arrangement of biomolecule template in the tissue of tooth surface. All of these sites will regulate the hydroxyapatite crystals arrange in parallel shape which the length of neonatal crystal is more close to beam crystal like nature enamel column. These studies provides reliable basic data for regeneration of enamel crystals.

\section{Conclusion}

In summary, the present work shows we are able to modifie the model of mineralization by phosphonized reaction which partly mimiced the physiological process of mineralization. Both of these neonatal structures are very similar in chemical composition and in structural dimensions to natural tooth enamel. The specific organic molecule model can be used as a potential effective crystal growth modifier. 


\section{Acknowledgement}

This work was supported by grant from National Natural Science Foundation of China: Controlled synthesis of enamel like hydroxyapatite on bio-modified macromolecule template (No. 81100786) and Key Technology Research and Development Program of the of SiChuan Province, China: Application on rapid mineralization of enamel superficial defect (2013SZ0020).

\section{References}

[1] Bolte M, Krefting ER, Clemen G, "Hard tissue of teeth and their calcium and phosphate content in Ambystoma mexicanum (Urodela: Ambystomatidae) ", Ann Anat, 1996 , 178,71-80.

[2] Aoba T, Moreno EC. "Changes in solubility of enamel mineral at various stages of porcine amelogenesis", Calcif Tissue Int. 1992, 50,266-272.

[3] Lippert F, Hara AT. "Fluoride dose-response of human and bovine enamel caries lesions under remineralizing conditions", Am J Dent. 2012, 25, 205-209.

[4] Iizuka J, Mukai Y, Taniguchi M, Mikuni-Takagaki Y, Ten Cate JM, Teranaka T. Chemical alteration by tooth bleaching of human salivary proteins that infiltrated subsurface enamel lesions-experimental study with bovine lesion model systems. Dent Mater J 2014,33:663-668.

[5] Amaral CM, Miranda ME, Correa DS, Silva EM. Sodium fluoride and casein phosphopeptide-amorphous calcium phosphate cream plus sodium fluoride efficacy in preventing enamel erosion in a simulated oral environment study model. Indian J Dent Res 2014;25:464-469.

[6] Wu D, Yang J, Li J, Chen L, Tang B, Chen X, Wu W, Li J. Hydroxyapatite-anchored dendrimer for in situ remineralization of human tooth enamel. Biomaterials 2013;34:5036-5047.

[7] Margolis HC, Kwak SY, Yamazaki H.Role of mineralization inhibitors in the regulation of hard tissue biomineralization: relevance to initial enamel formation and maturation. Front Physiol 2014;5:339-347.

[8] Guerriero G. "Putative Chitin Synthases from Branchiostoma floridae Show Extracellular Matrix-related Domains and Mosaic Structures”, Genomics Proteomics Bioinformatics, 2012, 10, 197-207.

[9] Wang J, Chen C. Chitosan-based biosorbents: modification and application for biosorption of heavy metals and radionuclides. Bioresour Technol 2014;160:129-141.

[10] Hayashi Y, Yamada S, Yanagi Guchi K, Koyama Z, Ikeda T, Chitosan and fish collagen as biomaterials for regenerative medicine, Adv Food Nutr Res 2012;65: 107-20.

[11] Oyane A, Kawashita M, Nakanishi K, Kokubo T, Minoda M, Miyamoto T, Nakamura T, "Bonelike apatite formation on ethylene-vinyl alcohol copolymer modified with silane coupling agent and calcium silicate solutions", Biomaterials,2003, 24, 1729-1735.

[12] Heras A, Rodríguez N.M, Ramos V.M, Agulló E, "N-methylene phosphonic chitosan: a novel soluble derivative", Carbohydr Polym, 2001,44, 1-8.

[13] Matsushita O, Koide T, Kobayashi R, Nagata K, Okabe A, "Substrate recognition by the collagen-binding domain of Clostridium histolyticum class I collagenase", J Biol Chem, 2001, 276, 8761-8770.

[14] Pabisch S, Wagermaier W, Zander T, Li C, Fratzl P. Imaging the nanostructure of bone and dentin through small- and wide-angle X-ray scattering. Methods Enzymol 2013,532:391-413. 
[15] Agrawal N, Shashikiran ND, Singla S, Ravi KS, Kulkarni VK. Effect of remineralizing agents on surface microhardness of primary and permanent teeth after erosion. J Dent Child (Chic) 2015,81:117-121.

[16] Wang Z, Shen Y, Haapasalo M, Wang J, Jiang T, Wang Y, Watson TF, Sauro S.Polycarboxylated microfillers incorporated into light-curable resin-based dental adhesives evoke remineralization at the mineral-depleted dentin. J Biomater Sci Polym Ed 2014,25:679-697.

[17] Sikirić MD, Füredi-Milhofer $H$. The influence of surface active molecules on the crystallization of biominerals in solution. Adv Colloid Interface Sci 2006,21:135-158.

[18] Mladenovic M, Arnone M, Fink RF, Engels B. Environmental effects on charge densities of biologically active molecules: do molecule crystal environments indeed approximate protein surroundings? J Phys Chem B 2009,113:5072-5082.

[19] Vaahtio M, Peltola T, Hentunen T, Ylänen H, Areva S, Wolke J, Salonen JI, " The properties of biomimetically processed calcium phosphate on bioactive ceramics and their response on bone cells", J Mater Sci Mater Med, 2006, 17, 1113-1125. 